\title{
Anterior-posterior differences in vertebrate segments: specification of trunk and tail somites in the zebrafish blastula
}

\author{
Scott A. Holley \\ Department of Molecular, Cellular and Developmental Biology, Yale University, New Haven, Connecticut 06520, USA
}

During vertebrate embryogenesis, the primary body axis grows posteriorly and is concomitantly segmented into somites, the precursors of the vertebral column, skeletal muscle, and dermis. The somites arise sequentially, with the anterior somites that give rise to the cervical vertebrae created early. The more posterior somites that become the thoracic, lumbar, and sacral vertebrae form at progressively later times. During the axis elongation period, the embryo must parse the somite precursors appropriately so that there are enough cells remaining to make the most posterior somites at the end of somitogenesis. How the embryo allocates these cells is not well understood. However, in this issue, Szeto and Kimelman (2006) address this question by showing that cells are specified to give rise to anterior trunk, posterior trunk, and tail somites. They find that this cell fate decision occurs surprisingly early in zebrafish development, prior to gastrulation, in response to nodal, $f g f$, and $\mathrm{bmp}$ signaling (Fig. 1; Szeto and Kimelman 2006). Their data link the processes of mesoderm induction and patterning with vertebrate segmentation and elucidate a mechanism by which the embryo reserves a somite precursor population for the formation of the more posterior body segments.

While the number of vertebrae varies greatly among different species (Richardson et al. 1998), somite number within a given species is remarkably consistent. The question arises, how does the embryo determine the appropriate somite number and size while the field of cells to be segmented continues to grow posteriorly? Experimentally manipulated Xenopus embryos, with large portions of the blastula physically removed, develop into embryos two-thirds smaller than normal, yet form the same number of segments and at the same rate as unmanipulated sibling embryos. Thus, the embryo "knows" the species-specific number of somites that it needs to generate and divides the available cell population accordingly; that is, there does not appear to be a physical con-

E-MAIL scott.holley@yale.edu; FAX (203) 432-5690.

Article is online at http://www.genesdev.org/cgi/doi/10.1101/gad.1453706. straint defining the number of cells in a given segment. In these smaller embryos, each segment consists of fewer cells than the somites of their normal-sized siblings (Cooke 1975). Furthermore, knypek; trilobite double mutants, which are much shorter than a wild-type zebrafish embryo due to a severe convergence extension defect, form somites only two cells in length, while zebrafish somites are normally five cells in length (Henry et al. 2000). Consideration of the regulative capacity of vertebrate segmentation led to the proposal that somitogenesis is controlled by a "clock and wavefront" whereby the clock represents a mechanism that causes the somite precursors to oscillate. Cells would only be able to form a segment during a brief period within each cycle of the somite clock. The wavefront represents the progression of tissue maturation and cell differentiation that sweeps head-to-tail along the primary axis of the embryo (Cooke and Zeeman 1975; Cooke 1998). In this model, a somite forms when the wavefront encounters a group of cells in the correct, permissive phase of the clock. Thus, somite length and rate of formation are dependent on the frequency of the clock/oscillator and the velocity of the wavefront. The regulative capacity of this mechanism allows the embryo to parse cells into segments at a rate that would retain enough cells to populate the most posterior somites. During the past 10 years, molecular evidence for both a clock and a wavefront has emerged (Pourquié 2003; Rida et al. 2004). However, Szeto and Kimelman (2006) find an additional mechanism by which the zebrafish embryo parses its supply of somite progenitors. They find that the anlagen of the anterior trunk, posterior trunk, and tail somites are specified before gastrulation, $5,13.5$, and $16.5 \mathrm{~h}$ before the onset of segmentation of each anlagen, respectively.

\section{nodal, fgf, and bmp signaling specify mesodermal precursor fates}

Generally speaking, nodal signaling induces and patterns the mesoderm, fgf and wnt signaling pattern and maintain mesodermal fates, while bmp signaling pat- 
Holley

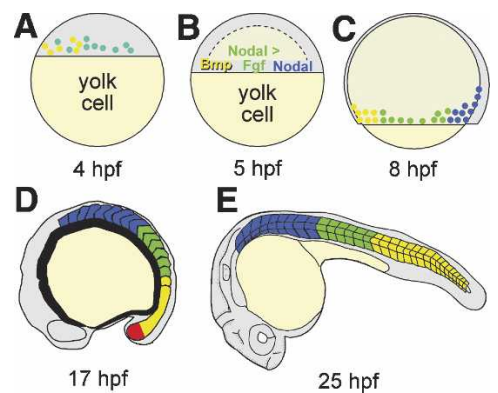

Figure 1. Specification of trunk and tail mesoderm in the zebrafish. (A) A fate map of the trunk (aqua) and tail somite (yellow) anlagen in a zebrafish embryo 4 hpf. Figure adapted with permission from Warga and Nusslein-Volhard (1999) (C) 1999 The Company of Biologists Ltd.). (B) By 5 hpf, the precursors for the anterior trunk, posterior trunk, and tail somites have been specified in response to Nodal, Nodal and Fgf, and Bmp ligands, respectively. $(C)$ By mid-gastrulation at $8 \mathrm{hpf}$, the anterior trunk and some of the posterior trunk MPCs converge toward the dorsal midline. The tail MPCs and some of the posterior trunk MPCs remain in a ventral-lateral position. Note that different MPC populations may be more mixed than depicted in $C$. In $A$, $B$, and $C$, dorsal/anterior is right and ventral/posterior is left. The embryo proper (gray) is at the animal pole, while the yolk cell is the vegetal pole. $(D)$ A mid-somitogenesis-stage embryo at $17 \mathrm{hpf}$. Anterior trunk somites (blue), posterior trunk somites (green), and presomitic mesoderm (yellow) are shown. The maturation or stem zone containing the mesodermal progenitor cells for the posterior tail somites is shown in red. (E) An embryo $25 \mathrm{hpf}$, at the end of somitogenesis. The tail somites are shown in yellow.

terns the ventral-posterior mesoderm (Schier and Talbot 2005; Kimelman 2006). Fate mapping of the zebrafish blastula shows that the dorsal mesoderm becomes the prechordal plate and notochord, while the more lateral mesoderm becomes the somites, nephros, and heart. The ventral mesoderm gives rise to tail somites and blood
(Kimmel et al. 1990; Warga and Nusslein-Volhard 1999). While these maps indicate the typical fate adopted by a cell in a particular location and time, the fate map does not indicate that the cells have been determined (i.e., irreversibly committed to a given fate) or specified (i.e., committed but still capable of altering their fate). Expanding on previous fate mapping studies, Szeto and Kimelman (2006) find that anterior trunk, posterior trunk, and tail somite anlagen are specified by nodal, fgf, and $b m p$ signaling during the late blastula.

In vertebrates, nodal signaling is required for the induction of both the mesoderm and endoderm. Nodals are members of the TGF $\beta$ superfamily and are represented by squint and cyclops in zebrafish (Schier and Talbot 2005). squint; cyclops double mutants lack all mesoderm and endoderm except for the tail somites (Feldman et al. 1998). Nodals signal through a serine/threonine kinase receptor complex that includes the type I and type II Activin receptors and the EGF-CFC coreceptor. This coreceptor is called one-eyed pinhead in zebrafish, and embryos lacking both the maternal and zygotic function of this gene (MZoep) resemble squint; cyclops double mutants (Fig. 2A; Gritsman et al. 1999). Before gastrulation, nodal signaling is highest at the margin, the equatorial portion of the embryo where the cells of the embryo proper are juxtaposed to the yolk cell. Due to activation of cyclops expression by squint dorsally, there is higher nodal signaling in the dorsoanterior part of the blastula (Dougan et al. 2003).

Fate mapping studies suggest that the progenitors of the trunk and tail somites are intermingled both during early development and within the tailbud that forms at the end of gastrulation (Fig. 1A; Kimmel et al. 1990; Kanki and Ho 1997; Warga and Nusslein-Volhard 1999). Szeto and Kimelman (2006) postulated that since only tail somites form in the absence of nodal signaling in MZoep embryos, that they could use this genetic back-
Figure 2. Notable differences between somites along the anterior-posterior axis. Green segments represent variability or ambiguity in the transitions, which are indicated in blue and yellow.
A

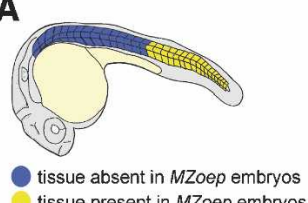
tissue present in MZoep embryos

D

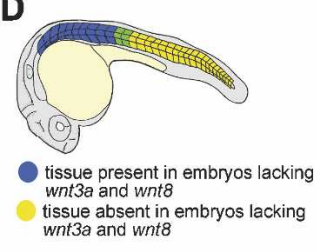

G

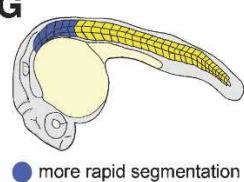

more rapid segmentatio slower segmentation
B

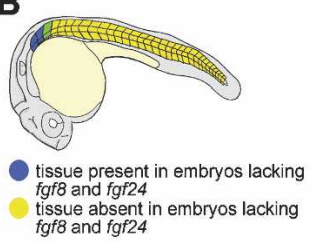

E

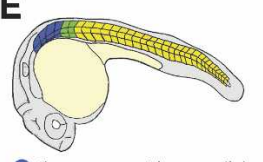

tissue present in oep;ntl double mutants tissue absent in oep;nt/ double mutant

H

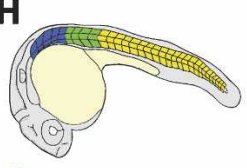

segmentation defective in integrin $\alpha .5$
and fibronectin 1 mutants and fibronectin 1 mutants segmentation defective in
notch pathway mutants

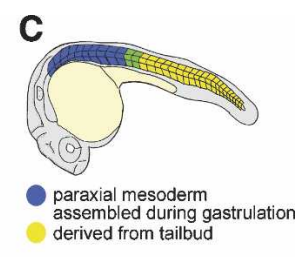

F

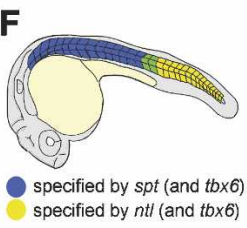

I

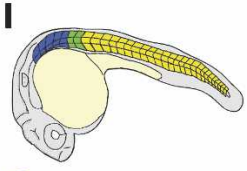

express sox 11a and nanos2 express neither sox 11 a 
ground to study the specification of trunk and tail mesodermal progenitor cells (MPCs). For this analysis, they used a cell transplantation technique to take cells from one embryo and place them in a host embryo of a different genetic background or stage of development. They found that if they transplanted MZoep cells from a lateblastula-stage embryo at $5 \mathrm{~h}$ post-fertilization (hpf) into a wild-type host at $5 \mathrm{hpf}$, the transplanted cells only populated the tail somites, whereas wild-type cells transplanted into a wild-type embryo populated all somites. These data indicate that MZoep cells have cell-autonomously adopted a tail MPC fate by $5 \mathrm{hpf}$. Heterochronic transplants of 5-hpf MZoep cells into a wild-type host embryo at 4 hpf show that the transplanted cells can populate the posterior trunk in addition to the tail. In contrast, if 4-hpf MZoep cells are transplanted into 5-hpf wild-type embryos, the donor cells only populate the tail. These two experiments indicate that the tail and trunk MPC fates are specified between 4 and $5 \mathrm{hpf}$, before the onset of gastrulation and $5 \mathrm{~h}$ before the beginning of somitogenesis (Fig. 1A,B). This specification signal(s) is missing in MZoep embryos but is present in wild-type embryos.

The trunk-promoting signal may consist of members of the $f g f$ family, at least three of which are expressed along the margin of the zebrafish blastula. fgf signaling promotes dorsal and dorsolateral fates in the zebrafish blastula. Moreover, $f g f$ expression is greatly reduced in MZoep embryos, meaning that $f g f$ is a good candidate for the trunk-inducing signal that is missing in these embryos (Mathieu et al. 2004). Indeed, Szeto and Kimelman (2006) show that injection of $f g f 4$ mRNA into a MZoep embryo and subsequent transplantation of these cells into a wild-type host result in donor cells populating the posterior trunk, somites 9-15. While this suggests that fgf is a trunk-promoting signal, these cells still do not populate the first nine somites, arguing that an additional signal is needed to specify anterior MPC fates. In fact, anterior trunk MPCs appear to require reception of Nodal. Szeto and Kimelman (2006) injected a constitutively active Nodal receptor into MZoep embryos and found that when transplanted into wild-type embryos, these donor cells populated the anterior nine somites. Thus, direct reception of Nodal is necessary for specification of anterior trunk (somites 1-9), while fgf promotes posterior trunk fates (somites 9-15). Later in development, after the MPC fates have been specified, fgf signaling is needed to maintain the population of both trunk and tail MPCs. Embryos lacking $f g f 8$ and $f g f 24$ retain only portions of the first three to four somites (Fig. 2B; Draper et al. 2003). Thus, these signaling pathways have distinct effects on the MPCs at different stages of development.

bmps are members of the Tgf $\beta$ superfamily and are necessary for specification of posterior tail and ventral fates such as somites and blood (Hammerschmidt and Mullins 2002). The Bmps are antagonized by the secreted inhibitors Chordin, Noggin, and Follistatin, which are expressed in the dorsal margin, called the shield or Spemann's Organizer in zebrafish and amphibians, respec- tively (Schier and Talbot 2005; Kimelman 2006). During gastrulation, dorsal and lateral mesendoderm undergo convergence toward the dorsal midline. The first 11-12 somites are largely derived of cells that have undergone some dorsal convergence and never passed through the posterior tailbud (Fig. 2C; Kanki and Ho 1997; Jülich et al. 2005). bmp signaling establishes a "no convergence zone" along the ventral margin and causes many of these cells to remain ventral, enter the tailbud, and ultimately contribute to the somites (Kanki and Ho 1997; Myers et al. 2002). Expression of the bmp inhibitors noggin, chordin, and follistatin is reduced in MZoep embryos (Gritsman et al. 1999; Ragland and Raible 2004). Thus, bmps are good candidates for a tail-promoting signal. Szeto and Kimelman (2006) injected bmp2b mRNA into MZoep embryos and transplanted these cells from 4-hpf donors to 4-hpf hosts. Uninjected MZoep cells may populate the posterior trunk, but the bmp2b-expressing cells displayed a stronger bias to populate the tail. These results are consistent with $b m p$ being a tail-promoting signal. Moreover, the trunk-promoting signal $f g f$ represses $b m p$ expression, suggesting that $f g f$ may specify trunk fates largely by inhibiting the tail-promoting bmp signal (Furthauer et al. 2004).

\section{wnt signaling and tail formation}

wnt signaling is also important for establishing and maintaining posterior, ventral mesodermal fates (Schier and Talbot 2005; Kimelman 2006). In zebrafish, wnt8 and wnt $3 a$ have partially redundant roles in tail formation, as morpholino inhibition of both genes results in a loss of tissue posterior to somites 10-12 (Fig. 2D). wnt8 and wnt $3 a$ appear to promote tail formation via caudal homologs, tbx 6 , and $f g f$, as expression of each is reduced in embryos lacking wnt8 and wnt3a (Szeto and Kimelman 2004; Shimizu et al. 2005; Thorpe et al. 2005). It is unclear if wnt signaling can recapitulate the tail-inducing activity of bmps in Szeto and Kimelman's (2006) MPC transplantation assay. However, it has been shown that bmp4, cylcops/nodal, and wnt8 can induce the formation of an ectopic tail when mRNAs encoding these genes are coinjected into animal blastomeres that would normally adopt ectodermal fates (Agathon et al. 2003). Notably in the mouse, wnt signaling is involved in both tail formation and segmentation (Aulehla et al. 2003).

\section{t-box genes and mesodermal patterning}

$t$-box genes are transcription factors that pattern the vertebrate mesoderm. In the zebrafish, no tail (the homolog of brachyuryl, spadetail, and tbx6 display a complex relationship in patterning the dorsal-ventral and anteriorposterior axes. spadetail, like nodal and $f g f$, is required for anterior and posterior trunk development. no tail is required for notochord and, like $b m p$ signaling, formation of tail somites. $t b x 6$ appears to act semiredundantly with both no tail and spadetail (Amacher and Kimmel 1998; Griffin et al. 1998; Griffin and Kimelman 2002, 2003; Goering et al. 2003). Despite the distinct pheno- 
types, these three $t$-box genes are expressed in largely overlapping domains. Due to multivarious regulatory interactions between the $t$-box genes, it has been suggested that a $t$-box code patterns the vertebrate mesoderm in a manner similar to the way in which the hox code specifies segmental identity (Goering et al. 2003).

The expression of these $t$-box genes is promoted by $f g f$ signaling, and, in turn, the $t$-box genes activate $f g f$ signaling in a positive feedback mechanism (Griffin et al. 1998; Draper et al. 2003; Griffin and Kimelman 2003). This feedback mechanism is important for maintaining mesodermal cell fates during gastrulation and axis elongation. During tail extension, $f g f$ signaling exists in a gradient with the high end in the posterior of the tailbud, the location of a maturation or stem zone that contains the MPCs that will give rise to the posterior trunk and tail (Fig. 1C; Dubrulle et al. 2001; Sawada et al. 2001; Draper et al. 2003; Dubrulle and Pourquié 2004). Cells in the maturation zone express no tail, spadetail, and tbx6 (Schulte-Merker et al. 1994; Griffin et al. 1998). Interestingly in the mouse, Tbx6 has been shown to activate the expression of the Notch ligand delta-like1, which is later involved in segmentation (Hofmann et al. 2004). After the MPCs leave the maturation zone and initiate the segmentation program, they begin to express a fourth $t$-box gene, $f_{s s} / t b \times 24$, which, as part of the segmentation wavefront, is required to interpret the segmentation clock, establish segment polarity, and promote morphological somite formation (van Eeden et al. 1996; Durbin et al. 2000; Holley et al. 2000; Holley and Takeda 2002; Nikaido et al. 2002). In summary, the $t$-box genes pattern the mesoderm along the anterior-posterior axis in a manner reflective of the anterior trunk, posterior trunk, and tail domains uncovered by Szeto and Kimelman (2006). Furthermore, the $t$-box genes have successive temporal roles in patterning, maintaining, and segmenting the mesoderm.

\section{Differences between anterior trunk and posterior trunk segmentation}

Somitogenesis occurs as blocks of cells at the anterior of the mesenchymal presomitic mesoderm (PSM) pinch off into epithelial somites. Studies of somitogenesis in mouse, chick, Xenopus, and zebrafish support a model in which somite formation is governed by a "somite clock," which causes oscillations in gene expression within the PSM. Propagation of these oscillations requires the notch pathway (mouse, zebrafish) and wnt3a (mouse). The oscillations are further modulated by the wavefront represented by fgf signaling (mouse, chick, zebrafish) and the $t$-box gene $f s s ; t b \times 24$ (zebrafish). Cessation of the oscillations in the anterior PSM leads to the establishment of polarity within each prospective segment and the onset of morphological somite formation. Transcriptional regulators of the mesp family along with notch/delta signaling are thought to establish segment polarity (Pourquié 2003; Rida et al. 2004). eph/ephrin signaling and integrin $\alpha 5 /$ fibronectin control the mesenchymal-to-epithelial transition during somite morphogen- esis (Durbin et al. 1998; Barrios et al. 2003; Jülich et al. 2005; Koshida et al. 2005).

In agreement with the findings of Szeto and Kimelman (2006), genetic and embryological experiments have uncovered several differences in the specification, formation, and differentiation of the anterior trunk, posterior trunk, and tail somites. Differences in the specification of the anterior paraxial mesoderm have been revealed by genetic experiments in mice and zebrafish. Mice mutant for either of the transcription factors mesogenin or $t b x 6$ form only the anterior trunk somites (Chapman and $\mathrm{Pa}$ paioannou 1998; Yoon and Wold 2000). Similarly, in zebrafish, Zoep; no tail double mutants lack all but the anterior trunk somites (Fig. 2E; Schier et al. 1997). Since no tail activates both spadetail and $f g f 8$ expression, the loss of posterior mesoderm in Zoep; no tail embryos is a likely due to a combined reduction of nodal, fgf, and t-box function (Draper et al. 2003; Griffin and Kimelman 2003). As discussed above, analysis of $t$-box genes in zebrafish has uncovered differences in the genetic hierarchy that specifies trunk and tail MPCs, with spadetail and $t b \times 6$ involved in specifying anterior and posterior trunk, while no tail and tbx6 specify tail MPCs (Fig. 2F; Kimmel et al. 1989; Griffin et al. 1998; Griffin and Kimelman 2002; Goering et al. 2003).

One consistent difference in anterior trunk somitogenesis observed in mice, zebrafish, and the cephalochordate amphioxus is the more rapid progression of the somite cycle relative to posterior somitogenesis (Tam 1981; Hanneman and Westerfield 1989; Schubert et al. 2001). In the zebrafish, the anterior six somites form every 20 min, while the 24 posterior somites form every $30 \mathrm{~min}$ (Fig. 2G; Hanneman and Westerfield 1989). In amphioxus, this temporal difference is even more extreme in that the anterior approximately eight somites form every hour but each subsequent somite cycle is $18 \mathrm{~h}$ (Schubert et al. 2001).

In zebrafish, fSS mutant embryos and embryos lacking both her1 and her 7 show segmentation defects along the entire body axis, indicating that there are common features in the genetic control of trunk and tail somitogenesis (van Eeden et al. 1996; Henry et al. 2002; Oates and Ho 2002). However, numerous genetic studies demonstrate that perturbation of notch signaling in mice, humans, and zebrafish, receptor tyrosine phosphatase $\psi$ in zebrafish, or mesp2 and wnt3a in mice leads to a segmentation defect in the posterior but not the anterior trunk somites with the defects occurring posterior to the fifth to ninth somite in zebrafish (Fig. $2 \mathrm{H}$; Rida et al. 2004). Accordingly, the deltaD mutant in zebrafish is called after eight, while the notch1a mutant is named deadly seven (van Eeden et al. 1996; Holley et al. 2000, 2002). Anterior trunk somitogenesis in zebrafish is also resistant to "dominant" perturbation of notch signaling via ectopic expression of an activated Notch, NICD, or a dominant-negative inhibitor of notch signaling, $\mathrm{X}-\mathrm{Su}(\mathrm{H})^{\mathrm{DBM}}$ (Wettstein et al. 1997; Takke and CamposOrtega 1999; Jülich et al. 2005). In contrast to perturbation of notch pathway function, zebrafish mutants for integrin $\alpha$, called before eight, and fibronectin1 affect 
the formation of only the first approximately seven somites (Fig. 2H; Jülich et al. 2005; Koshida et al. 2005). Zebrafish double mutants between the anterior and posterior specific somite mutants lack all segments. Interestingly, $f S S /+$ embryos or $f S S /+$; aei/deltaD/+; des/ notch1a/+ embryos display transient segmentation defects centered around the seventh to ninth somites. These observations suggest that the seventh to ninth somites represent a transition zone between anterior and posterior trunk somitogenesis in zebrafish (Jülich et al. 2005). It is currently unclear whether these differences in the segmentation program are related to the differences between anterior trunk, posterior trunk, and tail MPCs revealed by Szeto and Kimelman (2006).

In mice and humans, there are also mutations that more severely affect the differentiation of the anterior trunk somites. In the mouse, PDGFR $\alpha$ is thought to mediate signaling between the myotome and sclerotome, and mice mutant for this receptor show extensive fusion of the cervical vertebrae but milder defects in thoracic and lumbar vertebrae (Soriano 1997; Tallquist et al. 2000). This is similar to congenital human defects known as the Klippel-Feil syndrome in which the cervical vertebrae are fused but the rib cage is only moderately affected, if at all. The complementary, posterior segmentation defects in humans are a heterogeneous class including spondylocostal dysostosis, which can be caused by mutations in notch pathway genes /Clarke et al. 1998; Bulman et al. 2000; Pourquié and Kusumi 2001; Sparrow et al. 2006).

Paradoxically, despite these genetic differences between anterior trunk, posterior trunk, and tail somitogenesis, most genes transcribed in a segmental pattern are expressed in all somites. In zebrafish, there is no significant difference in the expression of deltaD, notch1a, integrina5, or fibronectin1 when comparing trunk and tail somites (Bierkamp and Campos-Ortega 1993; Dornseifer et al. 1997; Jülich et al. 2005; Koshida et al. 2005). There are a small number of genes that do show differential expression in the tail and trunk somites. For example, a nanos-related gene and sox11a are only expressed in the anterior trunk somites in zebrafish (Fig. 2I; de Martino et al. 2000; Jülich et al. 2005). The segmental expression of snail1a, Engrailed, and myoD arises simultaneously in the anterior trunk somites at the approximately six somite stage, while subsequent expression of these genes arises sequentially as each new somite is generated (Hatta et al. 1991; Ekker et al. 1992; Hammerschmidt and Nüsslein-Volhard 1993; Thisse et al. 1993; Weinberg et al. 1996; Jülich et al. 2005). However, none of these differentially expressed genes has been shown to function in the segmentation program. It is also unknown if the localized expression of these genes is related to the earlier specification of the anterior and posterior trunk.

\section{Summary}

The findings of Szeto and Kimelman (2006) begin to integrate our understanding of mesoderm patterning with vertebrate segmentation. Using a series of isochronic and heterchronic cell transplantation experiments, they show that the progenitors of the anterior trunk, posterior trunk, and tail somites are specified during a 1-h period in the zebrafish blastula by nodal, bmp, and $f g f$ signaling. Despite this patterning, fate mapping experiments indicate that these distinct MPC populations intermingle in the late blastula and within the posterior tailbud. At the cellular level, it is not clear how such a pattern is generated nor how cells "remember" when to exit the progenitor pool and enter the segmentation program. We also do not understand how the early specification of anterior trunk, posterior trunk, and tail MPCs relates to the control of segmentation, although the two processes share a dependence on $t$-box genes, $f g f$ signaling, and wnt signaling. The unresolved questions in the field involve larger issues fundamental to modern developmental biology and molecular medicine. Questions such as how signals are integrated, how one cell population can respond differently to the same signal at different times, and how cells decide to exit their progenitor stem cell niche and begin to differentiate need to be understood to both accurately model vertebrate development and to adroitly engineer stem cells.

\section{Acknowledgments}

I thank Bruce Draper, Gilbert Weidinger, and Randy Moon for clarifications, and Tim Brend, Dörthe Jülich, and Andrew Mara for critical comments on the manuscript. S.A.H. is supported by a grant from NICHD (HD045738-01A1).

\section{References}

Agathon, A., Thisse, C., and Thisse, B. 2003. The molecular nature of the zebrafish tail organizer. Nature 424: 448-452.

Amacher, S.L. and Kimmel, C.B. 1998. Promoting notochord fate and repressing muscle development in zebrafish axial mesoderm. Development 125: 1397-1406.

Aulehla, A., Wehrle, C., Brand-Saberi, B., Kemler, R., Gossler, A., Kanzler, B., and Herrmann, B.G. 2003. Wnt3a plays a major role in the segmentation clock controlling somitogenesis. Dev. Cell 4: 395-406.

Barrios, A., Poole, R.J., Durbin, L., Brennan, C., Holder, N., and Wilson, S.W. 2003. Eph/Ephrin signaling regulates the mesenchymal-to-epithelial transition of the paraxial mesoderm during somite morphogenesis. Curr. Biol. 13: 1571-1582.

Bierkamp, C. and Campos-Ortega, J.A. 1993. A zebrafish homologue of the Drosophila neurogenic gene Notch and its pattern of transcription during early embryogenesis. Mech. Dev. 43: 87-100.

Bulman, M.P., Kusumi, K., Frayling, T.M., McKeown, C., Garret, C., Lander, E.S., Krumlauf, R., Hattersley, A.T., Ellard, S., and Turnpenny, P.D. 2000. Mutations in the human delta homologue, DLL3, cause axial skeletal defects in spondylocostal dysostosis. Nat. Genet. 24: 438-441.

Chapman, D.L. and Papaioannou, V.E. 1998. Three neural tubes in mouse embryos with mutations in the T-box gene Tbx6. Nature 391: 695-697.

Clarke, R.A., Catalan, G., Diwan, A.D., and Kearsley, J.H. 1998. Heterogeneity in Klippel-Feil syndrome: A new classification. Pediatr. Radiol. 28: 967-974. 
Cooke, J. 1975. Control of somite number during morphogenesis of a vertebrate, Xenopus laevis. Nature 254: 196-199.

- 1998. A gene that resuscitates a theory-Somitogenesis and a molecular oscillator. Trends Genet. 14: 85-88.

Cooke, J. and Zeeman, E.C. 1975. A clock and wavefront model for control of the number of repeated structures during animal morphogenesis. J. Theor. Biol. 58: 455-476.

de Martino, S., Yan, Y.L., Jowett, T., Postlethwait, J.H., Varga, Z.M., Ashworth, A., and Austin, C.A. 2000. Expression of sox11 gene duplicates in zebrafish suggests the reciprocal loss of ancestral gene expression patterns in development. Dev. Dyn. 217: 279-292.

Dornseifer, P., Takke, C., and Campos-Ortega, J.A. 1997. Overexpression of a zebrafish homologue of the Drosophila neurogenic gene Delta perturbs differentiation of primary neurons and somite development. Mech. Dev. 63: 159-171.

Dougan, S.T., Warga, R.M., Kane, D.A., Schier, A.F., and Talbot, W.S. 2003. The role of the zebrafish nodal-related genes squint and cyclops in patterning of mesendoderm. Development 130: 1837-1851.

Draper, B.W., Stock, D.W., and Kimmel, C.B. 2003. Zebrafish fgf2 4 functions with fgf8 to promote posterior mesodermal development. Development 130: 4639-4654.

Dubrulle, J. and Pourquié, O. 2004. fgf8 mRNA decay establishes a gradient that couples axial elongation to patterning in the vertebrate embryo. Nature 427: 419-422.

Dubrulle, J., McGrew, M.J., and Pourquié, O. 2001. FGF signaling controls somite boundary position and regulates segmentation clock control of spatiotemporal Hox gene activation. Cell 106: 219-232.

Durbin, L., Brennan, C., Shiomi, K., Cooke, J., Barrios, A., Shanmugalingam, S., Guthrie, B., Lindberg, R., and Holder, N. 1998. Eph signaling is required for segmentation and differentiation of the somites. Genes \& Dev. 12: 3096-3109.

Durbin, L., Sordino, P., Barrios, A., Gering, M., Thisse, C., Thisse, B., Brennan, C., Green, A., Wilson, S., and Holder, N. 2000. Anteroposterior patterning is required within segments for somite boundary formation in developing zebrafish. Development 127: 1703-1713.

Ekker, M., Wegner, J., Akimenko, M.A., and Westerfield, M. 1992. Coordinate embryonic expression of three zebrafish engrailed genes. Development 116: 1001-1010.

Feldman, B., Gates, M.A., Egan, E.S., Dougan, S.T., Rennebeck, G., Sirotkin, H.I., Schier, A.F., and Talbot, W.S. 1998. Zebrafish organizer development and germ-layer formation require nodal-related signals. Nature 395: 181-185.

Furthauer, M., Van Celst, J., Thisse, C., and Thisse, B. 2004. Fgf signalling controls the dorsoventral patterning of the zebrafish embryo. Development 131: 2853-2864.

Goering, L.M., Hoshijima, K., Hug, B., Bisgrove, B., Kispert, A., and Grunwald, D.J. 2003. An interacting network of T-box genes directs gene expression and fate in the zebrafish mesoderm. Proc. Natl. Acad. Sci. 100: 9410-9415.

Griffin, K.J. and Kimelman, D. 2002. One-Eyed Pinhead and Spadetail are essential for heart and somite formation. Nat. Cell Biol. 4: 821-825.

2003. Interplay between FGF, One-Eyed Pinhead, and T-box transcription factors during zebrafish posterior development. Dev. Biol. 264: 456-466.

Griffin, K.J., Amacher, S.L., Kimmel, C.B., and Kimelman, D. 1998. Molecular identification of Spadetail: Regulation of zebrafish trunk and tail mesoderm formation by T-box genes. Development 125: 3379-3388.

Gritsman, K., Zhang, J., Cheng, S., Heckscher, E., Talbot, W.S., and Schier, A.F. 1999. The EGF-CFC protein One-Eyed Pinhead is essential for nodal signaling. Cell 97: 121-132.
Hammerschmidt, M. and Mullins, M.C. 2002. Dorsoventral patterning in the zebrafish: Bone morphogenetic proteins and beyond. Results Probl. Cell Differ. 40: 72-95.

Hammerschmidt, M. and Nüsslein-Volhard, C. 1993. The expression of a zebrafish gene homologous to Drosophila snail suggests a conserved function in invertebrate and vertebrate gastrulation. Development 119: 1107-1118.

Hanneman, E. and Westerfield, M. 1989. Early expression of acetyl-choline-sterase activity in functionally distinct neurons of the zebrafish. J. Comp. Neurol. 284: 350-361.

Hatta, K., Bremiller, R., Westerfield, M., and Kimmel, C.B. 1991. Diversity of expression of engrailed-like antigens in zebrafish. Development 112: 821-832.

Henry, C.A., Hall, L.A., Hille, M.B., Solnica-Krezel, L., and Cooper, M.S. 2000. Somite in zebrafish doubly mutant for knypek and trilobite form without internal mesenchymal cells or compaction. Curr. Biol. 10: 1063-1066.

Henry, C.A., Urban, M.K., Dill, K.K., Merlie, J.P., Page, M.F., Kimmel, C.B., and Amacher, S.L. 2002. Two linked hairy/ Enhancer of split-related zebrafish genes, herl and her7, function together to refine alternating somite boundaries. Development 129: 3693-3704.

Hofmann, M., Schuster-Gossler, K., Watabe-Rudolph, M., Aulehla, A., Herrmann, B.G., and Gossler, A. 2004. WNT signaling, in synergy with T/TBX6, controls Notch signaling by regulating Dll1 expression in the presomitic mesoderm of mouse embryos. Genes \& Dev. 18: 2712-2717.

Holley, S.A. and Takeda, H. 2002. Catching a wave: The oscillator and wavefront that create the zebrafish somite. Semin. Cell Dev. Biol. 13: 481-488.

Holley, S.A., Geisler, R., and Nüsslein-Volhard, C. 2000. Control of her1 expression during zebrafish somitogenesis by a Delta-dependent oscillator and an independent wave-front activity. Genes \& Dev. 14: 1678-1690.

Holley, S.A., Jülich, D., Rauch, G.J., Geisler, R., and NüssleinVolhard, C. 2002. her1 and the notch pathway function within the oscillator mechanism that regulates zebrafish somitogenesis. Development 129: 1175-1183.

Jülich, D., Geisler, R., and Holley, S.A. 2005. Integrin $\alpha 5$ and Delta/Notch signalling have complementary spatiotemporal requirements during zebrafish somitogenesis. Dev. Cell 8: 575-586.

Kanki, J.P. and Ho, R.K. 1997. The development of the posterior body in zebrafish. Development 124: 881-893.

Kimelman, D. 2006. Mesoderm induction: From caps to chips. Nat. Rev. Genet. 7: 360-372.

Kimmel, C.B., Kane, D.A., Walker, C., Warga, R.M., and Rothman, M.B. 1989. A mutation that changes cell movement and cell fate in the zebrafish embryo. Nature 337: 358-362.

Kimmel, C.B., Warga, R.M., and Schilling, T.F. 1990. Origin and organization of the zebrafish fate map. Development 108: 581-594.

Koshida, S., Kishimoto, Y., Ustumi, H., Shimizu, T., FurutaniSeiki, M., Kondoh, H., and Takada, S. 2005. Integrin $\alpha 5$-dependent fibronectin accumulation for maintenance of somite boundaries in zebrafish embryos. Dev. Cell 8: 587-598.

Mathieu, J., Griffin, K., Herbomel, P., Dickmeis, T., Strahle, U., Kimelman, D., Rosa, F.M., and Peyrieras, N. 2004. Nodal and Fgf pathways interact through a positive regulatory loop and synergize to maintain mesodermal cell populations. Development 131: 629-641.

Myers, D.C., Sepich, D.S., and Solnica-Krezel, L. 2002. Bmp activity gradient regulates convergent extension during zebrafish gastrulation. Dev. Biol. 243: 81-98.

Nikaido, M., Kawakami, A., Sawada, A., Furutani-Seiki, M., Takeda, H., and Araki, K. 2002. Tbx24, encoding a T-box 
protein, is mutated in the zebrafish somite-segmentation mutant fused somites. Nat. Genet. 31: 195-199.

Oates, A.C. and Ho, R.K. 2002. Hairy/E(spl)-related (Her) genes are central components of the segmentation oscillator and display redundancy with the Delta/Notch signaling pathway in the formation of anterior segmental boundaries in the zebrafish. Development 129: 2929-2946.

Pourquié, O. 2003. The segmentation clock: Converting embryonic time into spatial pattern. Science 301: 328-330.

Pourquié, O. and Kusumi, K. 2001. When body segmentation goes wrong. Clin. Genet. 60: 409-416.

Ragland, J.W. and Raible, D.W. 2004. Signals derived from the underlying mesoderm are dispensable for zebrafish neural crest induction. Dev. Biol. 276: 16-30.

Richardson, M.K., Allen, S.P., Wright, G.M., Raynaud, A., and Hanken, J. 1998. Somite number and vertebrate evolution. Development 125: 151-160.

Rida, P.C., Le Minh, N., and Jiang, Y.-J. 2004. A Notch feeling of somite segmentation and beyond. Dev. Biol. 265: 2-22.

Sawada, A., Shinya, M., Jiang, Y.-J., Kawakami, A., Kuroiwa, A., and Takeda, H. 2001. Fgf/MAPK signalling is a crucial positional cue in somite boundary formation. Development 128: 4873-4880.

Schier, A.F. and Talbot, W.S. 2005. Molecular genetics of axis formation in zebrafish. Annu. Rev. Genet. 39: 561-613.

Schier, A.F., Neuhauss, S.C.F., Helde, K.A., Talbot, W.S., and Driever, W. 1997. The one-eyed pinhead gene functions in mesoderm and endoderm formation in zebrafish and interacts with no tail. Development 124: 327-342.

Schubert, M., Holland, L.Z., Stokes, M.D., and Holland, N.D. 2001. Three amphioxus Wnt genes (AmphiWnt3, AmphiWnt5, and AmphiWnt6) associated with the tail bud: The evolution of somitogenesis in chordates. Dev. Biol. 240: 262-273.

Schulte-Merker, S., van Eeden, F.J.M., Halpern, M.E., Kimmel, C.B., and Nüsslein-Volhard, C. 1994. no tail (ntl) is the zebrafish homologue of the mouse T (Brachyury) gene. Development 120: 1009-1015.

Shimizu, T., Bae, Y.K., Muraoka, O., and Hibi, M. 2005. Interaction of Wnt and caudal-related genes in zebrafish posterior body formation. Dev. Biol. 279: 125-141.

Soriano, P. 1997. The PDGF $\alpha$ receptor is required for neural crest cell development and for normal patterning of the somites. Development 124: 2691-2700.

Sparrow, D.B., Chapman, G., Wouters, M.A., Whittock, N.V., Ellard, S., Fatkin, D., Turnpenny, P.D., Kusumi, K., Sillence, D., and Dunwoodie, S.L. 2006. Mutation of the LUNATIC FRINGE gene in humans causes spondylocostal dysostosis with a severe vertebral phenotype. Am. J. Hum. Genet. 78: $28-37$.

Szeto, D.P. and Kimelman, D. 2004. Combinatorial gene regulation by Bmp and Wnt in zebrafish posterior mesoderm formation. Development 131: 3751-3760.

— 2006. The regulation of mesodermal progenitor cell commitment to somitogenesis subdivides the zebrafish body musculature into distinct domains. Genes \& Dev. 20 (this issue).

Takke, C. and Campos-Ortega, J.A. 1999. her1, a zebrafish pairrule gene, acts downstream of Notch signaling to control somite development. Development 126: 3005-3014.

Tallquist, M.D., Weismann, K.E., Hellstrom, M., and Soriano, P. 2000. Early myotome specification regulates PDGFA expression and axial skeleton development. Development 127: 5059-5070.

Tam, P.P. 1981. The control of somitogenesis in mouse embryos. J. Embryol. Exp. Morphol. (Suppl.) 65: 103-128.
Thisse, C., Thisse, B., Schilling, T.F., and Postlethwait, J.H. 1993. Structure of the zebrafish snail1 gene and its expression in wild-type, spadetail and no tail mutant embryos. Development 119: 1203-1215.

Thorpe, C.J., Weidinger, G., and Moon, R.T. 2005. Wnt/ $\beta$ catenin regulation of the Sp1-related transcription factor sp5l promotes tail development in zebrafish. Development 132: 1763-1772.

van Eeden, F.J.M., Granato, M., Schach, U., Brand, M., FurutaniSeiki, M., Haffter, P., Hammerschmidt, M., Heisenberg, C.P., Jiang, Y.-J., Kane, D.A., et al. 1996. Mutations affecting somite formation and patterning in the zebrafish Danio rerio. Development 123: 153-164.

Warga, R.M. and Nusslein-Volhard, C. 1999. Origin and development of the zebrafish endoderm. Development 126: 827838 .

Weinberg, E.S., Allende, M.L., Kelly, C.S., Abdelhamid, A., Murakami, T., Anderman, P., Doerre, O.G., Grunwald, D.J., and Riggleman, B. 1996. Developmental regulation of zebrafish MyoD in wild-type, no tail and spadetail embryos. Development 122: 271-280.

Wettstein, D.A., Turner, D.L., and Kintner, C. 1997. The Xenopus homolog of Drosophila Suppressor of Hairless mediates Notch signaling during primary neurogenesis. Development 124: 693-702.

Yoon, J.K. and Wold, B. 2000. The bHLH regulator pMesogenin 1 is required for maturation and segmentation of paraxial mesoderm. Genes \& Dev. 14: 3204-3214. 


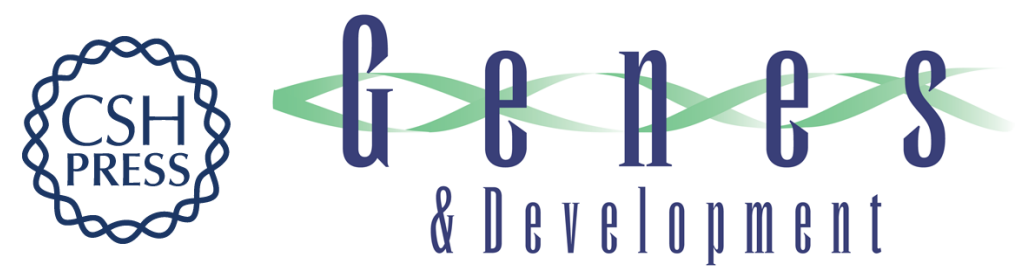

\section{Anterior-posterior differences in vertebrate segments: specification of trunk and tail somites in the zebrafish blastula}

Scott A. Holley

Genes Dev. 2006, 20:

Access the most recent version at doi:10.1101/gad.1453706

$\begin{array}{cl}\text { Related Content } & \begin{array}{l}\text { The regulation of mesodermal progenitor cell commitment to somitogenesis } \\ \text { subdivides the zebrafish body musculature into distinct domains } \\ \text { Daniel P. Szeto and David Kimelman } \\ \text { Genes Dev. UNKNOWN, 2006 20: 1923-1932 }\end{array} \\ \text { References } & \begin{array}{l}\text { This article cites 71 articles, 34 of which can be accessed free at: } \\ \text { http://genesdev.cshlp.org/content/20/14/1831.full.html\#ref-list-1 } \\ \text { Articles cited in: } \\ \text { http://genesdev.cshlp.org/content/20/14/1831.full.html\#related-urls }\end{array} \\ \text { License } & \begin{array}{l}\text { Receive free email alerts when new articles cite this article - sign up in the box at the top } \\ \text { right corner of the article or click here. }\end{array}\end{array}$

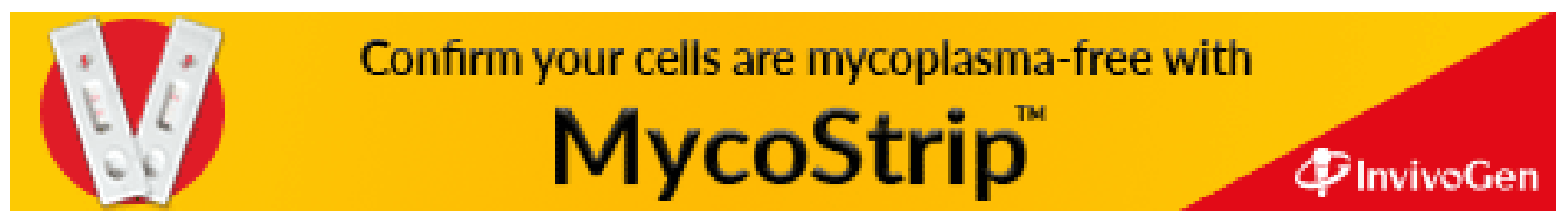

